\title{
Security Analysis of Niu et al. Authentication and Ownership Management Protocol
}

\author{
Nasour Bagheri, Masoumeh Safkhani and Hoda Jannati
}

\begin{abstract}
Over the past decade, besides authentication, ownership management protocols have been suggested to transfer or delegate the ownership of RFID tagged items. Recently, Niu et al. have proposed an authentication and ownership management protocol based on 16-bit pseudo random number generators and exclusive-or operations which both can be easily implemented on low-cost RFID passive tags in EPC global Class-1 Generation-2 standard. They claim that their protocol offers location and data privacy and also resists against desynchronization attack. In this paper, we analyze the security of their proposed authentication and ownership management protocol and show that the protocol is vulnerable to secret disclosure and desynchronization attacks. The complexity of most of the attacks are only two runs of the protocol and the success probability of the attacks are almost 1.
\end{abstract}

Index Terms-RFID, ownership transfer, ownership delegation, secret disclosure attack, desynchronization attack.

\section{INTRODUCTION}

Radio Frequency IDentifiction (RFID) is a wireless identification technology which contains tags, readers and servers and works using radio waves. Tag is a microchip which connects to the objects and the reader can read or modify the information of tags. Complex operations can be done in the servers and also more information about tags and readers are stored in them. There are three kinds of tags which are passive, active and semi-passive. Passive tags have no batteries and receive their energies from the reader, while the active tags have internal batteries which lead to increase their cost. Semipassive tags are between passive and active tags, i.e., they have a small battery for some of their functionalities and the required energy for other functionalities is obtained form the reader [15].

Electronic Product Code Class-1 Generation-2 (or in brief EPC-C1G2) [6], [12] is one of the important standards related to passive tags which supports only Cyclic Redundancy Check (CRC) functions, Pseudo Random Number Generator (PRNG) functions and lightweight operations such as AND, OR, XOR and etc. Due to the widespread use of passive tags, many EPCC1G2 complaint protocols such as authentication [16], [29], [18], [5], [17], [19], ownership transfer [9], tag search [27], [28], distance bounding protocols [10], grouping proof [26], [21], [20] and etc [14] have been designed. There also are

Nasour Bagheri is with the Department of Electrical Engineering, Shahid Rajaee Teachers Training University, Tehran, Iran (email: NBagheri@srttu.edu).

Masoumeh Safkhani is with the Department of Computer Engineering, Shahid Rajaee Teachers Training University, Tehran, Iran (email: Safkhani@srttu.edu).

Hoda Jannati is with the School of Computer Science, Institute for Research in Fundamental Sciences (IPM), Tehran, Iran (email: hodajannati@ipm.ir). many reports on vulnerabilities of these protocols against different attacks [13], [23], [4], [3], [30], [1], [8], [22], [2], [7], [25]. All of these efforts show that designing a secure protocol in the framework of EPC-C1G2 is not a straighted forward task and we still need secure protocols in this area. Recently, in response to this need, in [11], Niu et al. presented a mutual authentication and ownership management protocol including ownership transfer and ownership delegation in order to provide location and data privacy in EPC-C1G2 passive tags. Their protocol relies only on pseudo random number generators and exclusive-or operations for execution. Both operations are easily implemented on low-cost RFID passive tags that comply with EPC-C1G2 standard. Niu et al. claim that their protocol provides location privacy, backward privacy, forward privacy and also suitable security against replay attack, desynchronization attack and windowing.

In this paper, we analyze the security of the authentication and ownership management protocol proposed by Niu et $a l$. and show that unfortunately their security claims do not hold. In particular, their protocol is vulnerable against secret disclosure and desynchronization attacks. In fact, this paper shows that this need is still unmet.

The rest of this paper is arranged as follows: In Section II, we review authentication and ownership management protocol proposed by Niu et al. Secret disclosure and the desynchronization attacks against the protocol are presented in Sections III and IV respectively and finally Section V concludes the paper.

\section{ReView of NiU et al. Authentication AND OWNership MANAGEMENT PROTOCOL}

There are four types of players in the protocol proposed by Niu et al. [11]:

1) A trusted third party $T T P$

2) An RFID tag $T$

3) An old owner (reader $R_{I D 1}$ )

4) A new owner (reader $R_{I D 2}$ )

It must be noted that Niu et al. have assumed all the protocol parameters are 96 bits to preserve compatibility to EPC standard and all 96-bit parameters are broken into six 16-bit words because of convenience of the protocol implementation [11]. To prevent desynchronization attack, they also have assumed that the reader and the tag both should maintain their old and current pseudonyms and keys.

In the following, we begin with an overview of the system notations shown in Table I, then their protocol is described in three phases. 
TABLE I

NOTATIONS UTILIZED TO FORMULATE NIU et al. PROTOCOL

\begin{tabular}{|c|c|}
\hline Symbol & Description \\
\hline$E P C$ & The unique and static electronic product code of the tag $T$ \\
\hline$I D S$ & The pseudonym of the tag $T$ \\
\hline$R_{I D_{i}}$ & The identifier of $i^{t h}$ reader \\
\hline$K^{D_{2}}$ & A secret key which is shared between the tag $T$ and its owner \\
\hline$K_{M}$ & $\begin{array}{l}\text { A master key which is shared between the tag } T \text { and its owner } \\
\text { (the owner of the tag } T \text { with } K_{M} \text { is able to modify the key } K \text { ) }\end{array}$ \\
\hline $\begin{array}{l}K_{T T P} \\
W(j)\end{array}$ & A secret key which is shared between the tag $T$ and $T T P$ \\
\hline $\begin{array}{l}W(\imath) \\
P R N G(.)\end{array}$ & $\begin{array}{l}\text { A 16-bit of } W \\
\text { A } 16 \text {-bit pseudo random number generator }\end{array}$ \\
\hline $\operatorname{Per}(X, Y)$ & $\begin{array}{l}\text { The permutation of } X=x_{1} x_{2} \ldots x_{n} \text { according to } Y=y_{1} y_{2} \ldots y_{n}\left(x_{i}, y_{i} \in\{0,1\} \text {, for } 0 \leq i \leq n\right) \text { as } \\
\operatorname{Per}(X, Y)=x_{k_{1}} x_{k_{2}} \ldots x_{k_{m}} x_{k_{n}} x_{k_{n-1}} \ldots x_{k_{m+2}} x_{k_{m+1}} \text { where } m(0 \leq m \leq n) \text { is the hamming weight of } Y \text {, } \\
\text { so that } y_{k_{1}}=y_{k_{2}}=\ldots=y_{k_{m}}=1 \text { and } y_{k_{m+1}}=y_{k_{m+2}}=\ldots=y_{k_{m}}=0 \text { for } 1 \leq k_{1}<k_{2}<\ldots<k_{m} \leq n \\
\text { and } 1 \leq k_{m+1}<k_{m+2}<\ldots<k_{n} \leq n\end{array}$ \\
\hline
\end{tabular}

\section{Mutual Authentication Phase:}

In the mutual authentication phase of Niu et al. protocol the reader $R_{I D 1}$ (the old owner of the tag $T$ ) authenticates the tag $T$ before the reader $R_{I D 1}$ delegates the ownership of the tag $T$ to the reader $R_{I D 2}$. This phase of the protocol is shown in Figure 1 and described below:

1) To start this phase of the protocol, the reader $R_{I D 1}$ (the old owner of the tag $T$ ) generates two random numbers $r n d_{1}$ and $r n d_{2}$. Then, it computes $A(i)=r n d_{1}(i) \oplus$ $P R N G\left(K(i) \oplus R_{I D 1}(i)\right) \oplus P R N G\left(K(i) \oplus R_{I D 2}(i)\right)$, $B(i)=\operatorname{rnd}_{2}(i) \oplus \operatorname{PRNG}\left(\operatorname{rnd}_{1}(i) \oplus K(i)\right)$ and $C(i)=P R N G\left(\operatorname{rnd}_{1}(i) \oplus R_{I D 1}(i)\right) \oplus$ $P R N G\left(\operatorname{rnd}_{2}(i) \oplus R_{I D 2}(i)\right)$ for $i=1, \ldots, 6$.

2) The reader $R_{I D 1}$ sends $A, B$ and $C$ to the tag $T$.

3) After receiving the messages $A, B$ and $C$ from the reader $R_{I D 1}$, the tag $T$ computes $r n d_{1}(i)=A(i) \oplus$ $P R N G\left(K(i) \oplus R_{I D 1}(i)\right) \oplus P R N G\left(K(i) \oplus R_{I D 2}(i)\right)$, $r n d_{2}(i)=B(i) \oplus \operatorname{PRNG}\left(\operatorname{rnd}_{1}(i) \oplus K(i)\right)$ and $C^{\prime}(i)=\operatorname{PRNG}\left(\operatorname{rnd}_{1}(i) \oplus R_{I D 1}(i)\right) \oplus$ $P R N G\left(\operatorname{rnd}_{2}(i) \oplus R_{I D 2}(i)\right)$ for $i=1, \ldots, 6$. Then, the $\operatorname{tag} T$ verifies whether $C^{\prime} \stackrel{?}{=} C$ is or not. In the case of equality, the tag $T$ authenticates the reader $R_{I D 1}$, updates $K(i)$ and $I D S(i)$ as $K^{*}(i)=\operatorname{Per}\left(\operatorname{rnd}_{1}(i), K(i)\right) \oplus K((i+1) \bmod 6)$ and $I D S^{*}(i)=\operatorname{Per}\left(\operatorname{rnd}_{2}(i), K(i)\right) \oplus K(i)$ respectively, and computes $D(i)=P R N G\left(K^{*}(i) \oplus I D S^{*}(i)\right)$ for $i=1, \ldots, 6$.

4) The tag $T$ sends $D$ to the reader $R_{I D 1}$.

5) After receiving the message $D$ from the tag $T$, the reader $R_{I D 1}$ computes $K^{*}(i)=$ $\operatorname{Per}\left(\operatorname{rnd}_{1}(i), K(i)\right) \oplus K((i+1) \bmod 6)$, $I D S^{*}(i)=\operatorname{Per}\left(\operatorname{rnd}_{2}(i), K(i)\right) \oplus K(i)$ and $D^{\prime}(i)=P R N G\left(K^{*}(i) \oplus I D S^{*}(i)\right)$ for $i=1, \ldots, 6$. Then, it verifies whether $D^{\prime} \stackrel{?}{=} D$ is or not. In the case of equality, it authorizes the tag $T$ and updates $K$ and $I D S$ as $K^{*}$ and $I D S^{*}$ respectively.

\section{Ownership Delegation Phase:}

In ownership delegation phase of the protocol, the reader $R_{I D 1}$ (which is the old owner of the tag $T$ ) wants to delegate all its rights over the $\operatorname{tag} T$ to the reader $R_{I D 2}$ by using the parameter called ticket. The old owner $R_{I D 1}$ and the tag $T$ both compute ticket $=K_{M} \oplus E P C \oplus r n d_{1} \oplus r n d_{2}$. Then, the reader $R_{I D 1}$ sends ticket, EPC, IDS and $K$ through a secure channel to the reader $R_{I D 2}$ (the new owner $R_{I D 2}$ of the tag $T$ ). Ownership delegation steps shown in Figure 2 are as follows:

1) The reader $R_{I D 2}$ sends its identification $R_{I D 2}$ and a Query command to the tag $T$.

2) The tag $T$ sends its $I D S$ to the reader $R_{I D 2}$.

3) The reader $R_{I D 2}$ generates one random number $r n d_{3}$, computes $E(i)=\operatorname{rnd}_{3}(i) \oplus$ $P R N G\left(K(i) \oplus R_{I D 2}(i)\right) \oplus \operatorname{PRNG}(K(i)) \quad$ and $F(i)=P R N G\left(\right.$ ticket $\left.(i) \oplus \operatorname{rnd}_{3}(i)\right)$ for $i=1, \ldots, 6$.

4) The reader $R_{I D 2}$ sends $E$ and $F$ to the tag $T$.

5) After receiving the messages $E$ and $F$ from the reader $R_{I D 2}$, the tag $T$ computes $\operatorname{rnd}_{3}(i)=$ $E(i) \oplus P R N G\left(K(i) \oplus R_{I D 2}(i)\right) \oplus P R N G(K(i))$ and $F^{\prime}(i)=P R N G\left(\right.$ ticket $\left.(i) \oplus \operatorname{rnd}_{3}(i)\right)$ for $i=1, \ldots, 6$. Then, the $\operatorname{tag} T$ verifies whether $F^{\prime} \stackrel{?}{=} F$. In the case of equality, the $\operatorname{tag} T$ authenticates the new owner $R_{I D 2}$ and updates $K(i)$ and $I D S(i)$ as $K^{*}(i)=\operatorname{Per}\left(\operatorname{rnd}_{3}(i), K(i)\right) \oplus K((i+1) \bmod 6)$ and $I D S^{*}(i)=\operatorname{Per}\left(r n d_{3}(i), K(i)\right) \oplus K(i)$ respectively as well as computing $G(i)=P R N G\left(K^{*}(i) \oplus I D S^{*}(i)\right)$ for $i=1, \ldots, 6$.

6) The $\operatorname{tag} T$ sends $G$ to the reader $R_{I D 2}$.

7) After receiving the message $G$ from the tag $T$, the reader $R_{I D 2}$ computes $K^{*}(i)=$ $\operatorname{Per}\left(\operatorname{rnd}_{3}(i), K(i)\right) \oplus K((i+1) \bmod 6)$ and $I D S^{*}(i)=\operatorname{Per}\left(\operatorname{rnd}_{3}(i), K(i)\right) \oplus K(i)$ and $G^{\prime}(i)=\operatorname{PRNG}\left(K^{*}(i) \oplus I D S^{*}(i)\right)$ for $i=1, \ldots, 6$. 


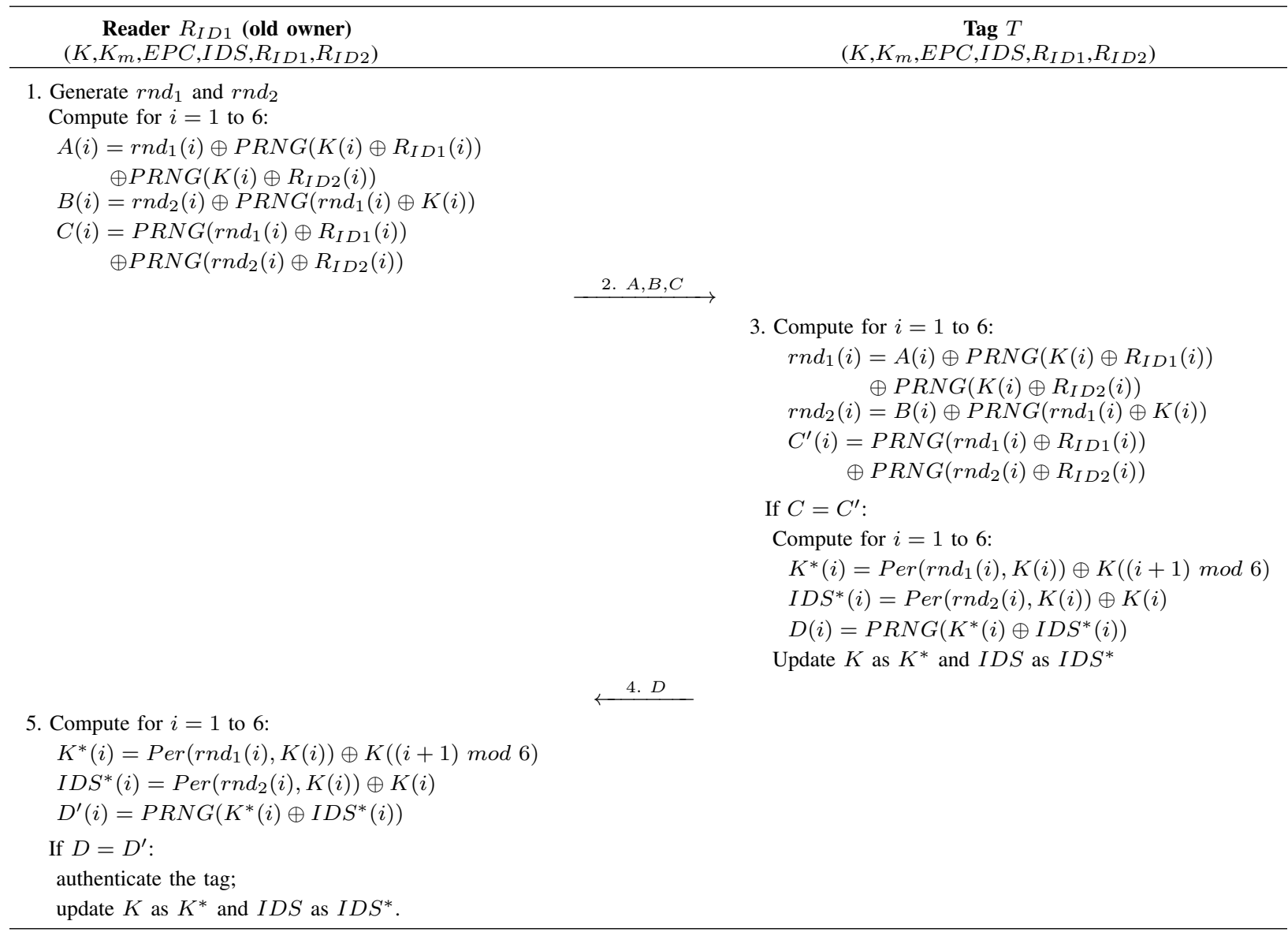

Fig. 1. Mutual authentication phase of Niu et al. authentication and ownership management protocol [11]

Then, it verifies whether $G^{\prime} \stackrel{?}{=} G$ is or not. In the case of equality, it authorizes the tag $T$ and updates $K$ and $I D S$ as $K^{*}$ and $I D S^{*}$ respectively.

\section{Complete Ownership Transfer Phase:}

The above mentioned ownership delegation transfer has not the property of the backward privacy since the old owner $R_{I D 1}$ holds the same values shared between the new owner $R_{I D 2}$ and the tag $T$. In order to address this pitfall, Niu et al. have proposed the complete ownership phase by using TTP in which all its rights over the tag $T$ are transferred to the reader $R_{I D 2}$ as a new owner. This phase is shown in Figure 3 and described below:

1) $T T P$ generates a random number $\mathrm{rnd}_{4}$, calculates $H(i)=\operatorname{rnd}_{4}(i) \oplus \operatorname{PRNG}\left(K_{T T P}(i)\right)$, $L(i)=\operatorname{PRNG}\left(K_{M}(i) \oplus \operatorname{rnd}_{4}(i)\right) \quad$ and $K_{M}^{*}(i)=\operatorname{PRNG}\left(\operatorname{Per}\left(K_{M}, \operatorname{rnd}_{4}(i)\right)\right.$ for $i=1$ to 6 . Then, TTP updates $K_{M}$ as $K_{M}^{*}$.

2) $T T P$ sends $K_{M}^{*}$ to the reader $R_{I D 2}$ (the new owner). It also sends $H$ and $L$ to the $\operatorname{tag} T$.

3) Once the tag $T$ received the messages $H$ and $L$, it retrieves $\operatorname{rnd}_{4}(i)$ as $H(i) \oplus \operatorname{PRNG}\left(K_{T T P}(i)\right)$ and computes $L^{\prime}(i)=\operatorname{PRNG}\left(K_{M}(i) \oplus \operatorname{rnd}_{4}(i)\right)$ for $i=1, \ldots, 6$. Then, the tag $T$ verifies whether
$L^{\prime} \stackrel{?}{=} L$. In the case of equality, it updates $K_{M}(i)$ as $K_{M}^{*}(i)=P R N G\left(\operatorname{Per}\left(K_{M}(i), \operatorname{rnd}_{4}(i)\right)\right)$ for $i=1$ to 6.

4) New owner $R_{I D 2}$ and the tag $T$ go to mutual authentication phase. If mutual authentication succeeds, the ownership transfer has successfully been done.

\section{SECRET Disclosure ATtACK ON NiU et al. AUTHENTICATION AND MANAGEMENT PROTOCOL}

In this section, we show that it is possible to disclose secret parameters in Niu et al. authentication and management protocol efficiently. The main observation is that in this protocol the 96-bit parameters are divided into 16-bit strings and messages are generated using a 16-bit PRNG. On the other hand, several related works have shown that it is hard to achieve high security using small components [24]. Based on this observation, we present an attack to disclose secret parameters in this protocol.

In an off-line phase of the attack, the adversary creates a table $T B$ and for $0 \leq x<2^{16}$ stores $(x, P R N G(x))$ in $T B$. Hence, given $T B$ and $P R N G(x)$, it is possible to determine possible values of $x$. We use $(A, B, C)_{r_{1}^{j}, r_{2}^{j}}^{K^{j}}$ to show the messages based on the secret key $K=K^{j}$ and random values $r n d_{1}=r_{1}^{j}$ and $r n d_{2}=r_{2}^{j}$. The secret disclosure attack works as follows: 


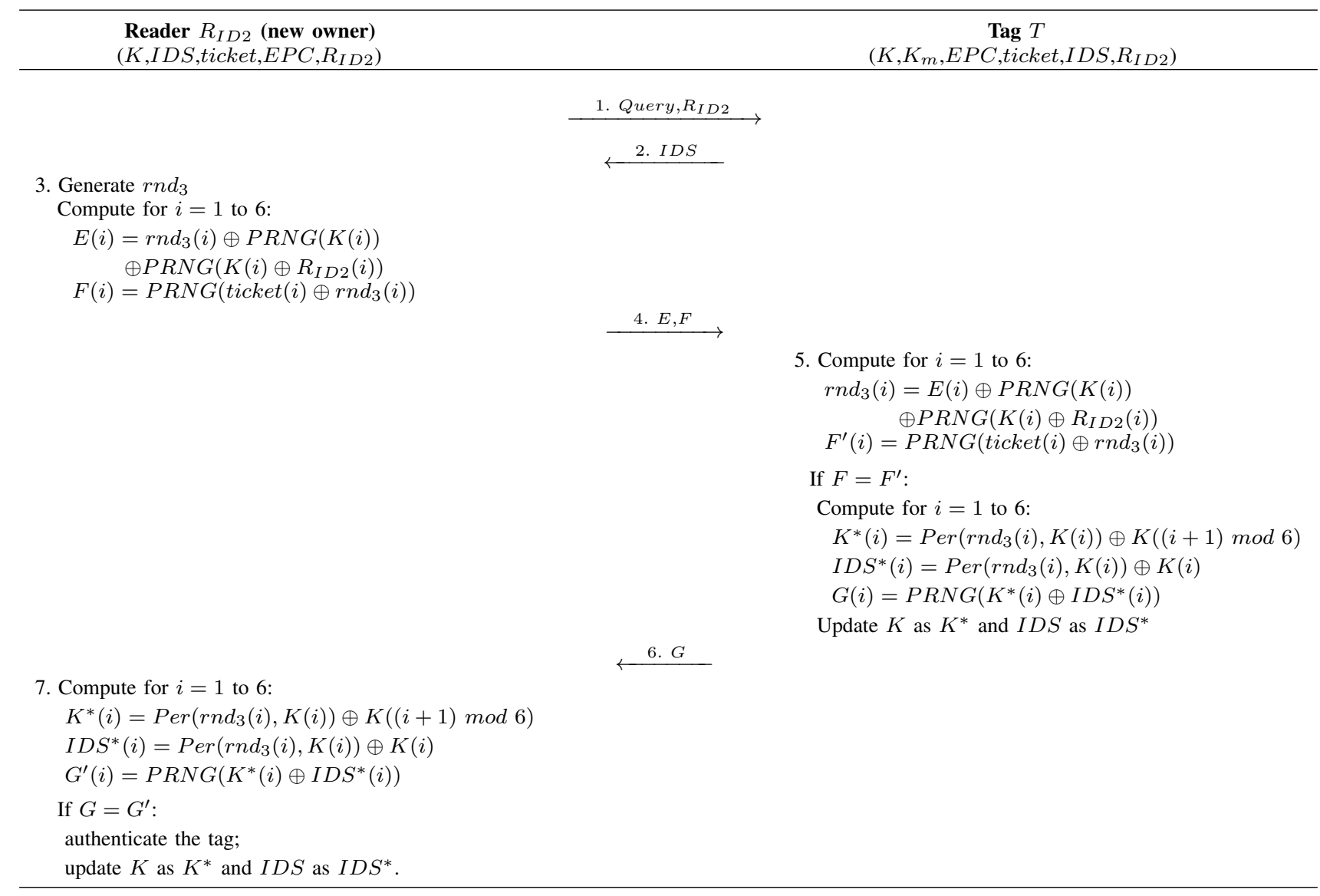

Fig. 2. Ownership delegation phase of Niu et al. authentication and ownership management protocol [11]

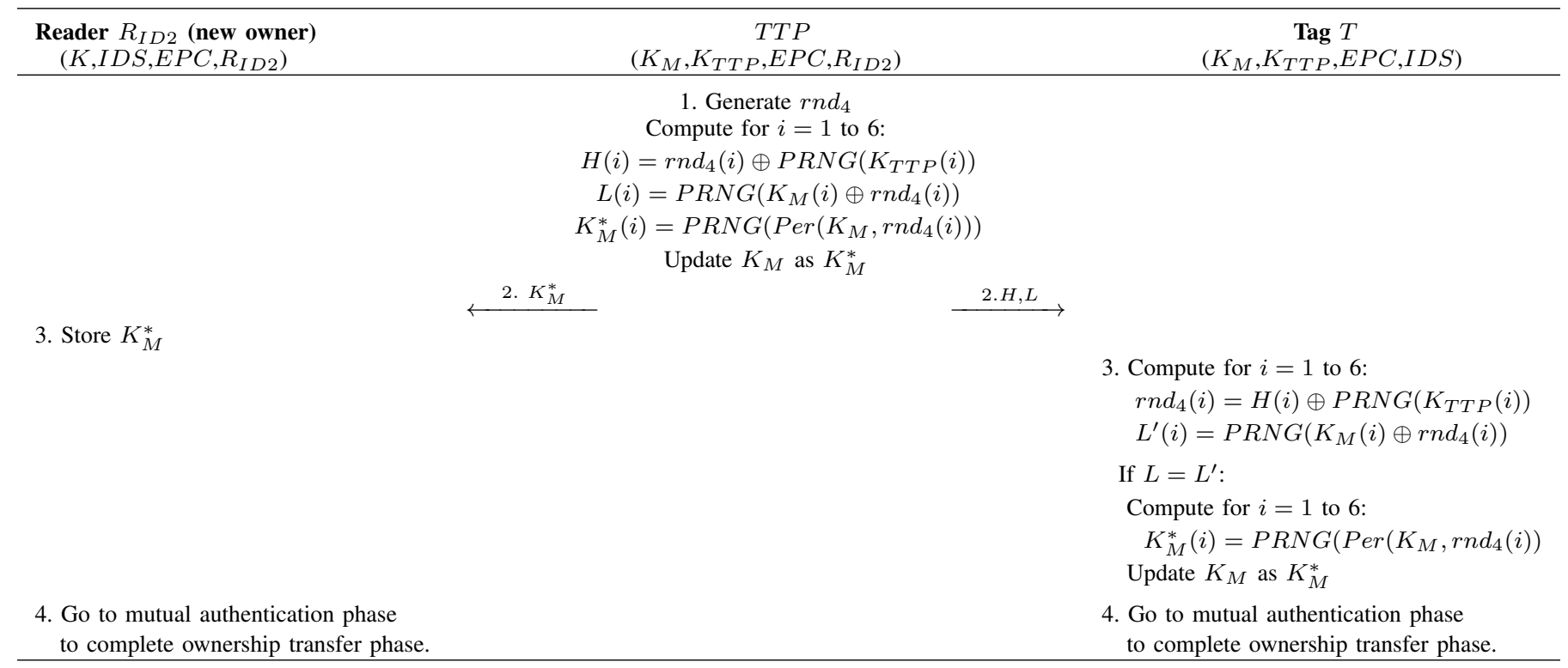

Fig. 3. Complete ownership transfer phase of Niu et al. authentication and ownership management protocol [11] 
1) Assume that the current state of the tag $T$ and the reader $R_{I D 1}$ is $\left(K^{0}, I D S^{0}, K^{1}, I D S^{1}\right)$ where $K^{0}$ and $I D S^{0}$ are the old key and pseudonym as well as $K^{1}$ and $I D S^{1}$ are the current key and pseudonym of the $\operatorname{tag} T$. The adversary also has a table $T B$ include $(x, P R N G(x))$ for $0 \leq x<2^{16}$.

2) In the mutual authentication phase, the reader $R_{I D 1}$ (old owner of the tag $T)$ sends $(A, B, C)_{r_{1}^{1}, r_{2}^{1}}^{K^{1}}$ to the tag $T$.

3) The tag $T$ updates its state to $\left(K^{1}, I D S^{1}, K^{2}, I D S^{2}\right)$ and sends $D(i)=P R N G\left(K^{2}(i) \oplus I D S^{2}(i)\right)$ to the reader $R_{I D 1}$ for $i=1, \ldots, 6$.

4) The adversary eavesdrops $(A, B, C)_{r_{1}^{1}, r_{2}^{1}}^{K^{1}}$ and $D$. The adversary also determines possible values of $K^{2}(i) \oplus$ $I D S^{2}(i)$, for $i=1, \ldots, 6$, using $T B$ and $D$.

5) In the ownership delegation phase, the reader $R_{I D 2}$ (new owner of the tag $T$ ) sends its identification $R_{I D 2}$ and a Query command to the tag $T$.

6) The tag $T$ sends its $I D S^{2}$ to the reader $R_{I D 2}$.

7) The adversary eavesdrops $I D S^{2}$ and determines possible values of $K^{2}$, given the result of step 4 .

8) The reader $R_{I D 2}$ generates a random number $r_{3}^{2}$ and computes $E(i)=r_{3}^{2}(i) \oplus P R N G\left(K^{2}(i) \oplus R_{I D 2}(i)\right) \oplus$ $P R N G\left(K^{2}(i)\right)$ and $F(i)=P R N G\left(\right.$ ticket $\left.(i) \oplus r_{3}^{2}(i)\right)$ for $i=1, \ldots, 6$ and sends $E$ and $F$ to the tag $T$, where ticket $=K_{M} \oplus E P C \oplus r_{1}^{1} \oplus r_{2}^{1}$.

9) The adversary eavesdrops $E$ and $F$ and determines possible values of $r_{3}^{2}$ and ticket using $T B$.

10) Once the $\operatorname{tag} T$ received the messages $E$ and $F$, it authenticates the new owner $R_{I D 2}$ and updates $K(i)$ and $I D S(i)$ as below:

$$
\begin{aligned}
& K^{3}(i)=\operatorname{Per}\left(r_{3}^{2}(i), K^{2}(i)\right) \oplus K^{2}((i+1) \bmod 6) ; \\
& I D S^{3}(i)=\operatorname{Per}\left(r_{3}^{2}(i), K^{2}(i)\right) \oplus K^{2}(i) .
\end{aligned}
$$

11) The tag $T$ computes $G_{i}=P R N G\left(K^{3}(i) \oplus I D S^{3}(i)\right)$ for $i=1, \ldots, 6$ and sends it to the reader $R_{I D 2}$.

12) The adversary eavesdrops $G$ and uses $T B$ to determine possible values of $K^{3}(i) \oplus I D S^{3}(i)$ for $i=1$ to 6 .

Given information extracted in steps 7 and 9, the adversary have some possible values of $K^{2}(i)$ and $r_{3}^{2}(i)$ for $i=1, \ldots, 6$. On the other hand, given $K^{2}(i), K^{2}((i+1) \bmod 6)$ and $r_{3}^{2}(i)$, it is possible to determine $K^{3}(i)$ and $I D S^{3}(i)$. Hence, given the extracted information from step 12 of the attack, it is possible for the adversary to filter the wrong guesses for the extracted $K^{2}(i)$ and $r_{3}^{2}(i)$. Following the given attack, the adversary can extract the tag's secret parameters, i.e., $K^{2}, I D S^{2}, K^{3}$ and $I D S^{3}$. The major complexity of the attack is eavesdropping one run of the protocol and $2^{16}$ calls to a $P R N G$ function in an off-line mode. For simulation purposes, the proposed attack is performed 1000 times over Niu et al. protocol. Simulation results show that the success probability of the attacker is almost 1 .

Moreover, each tag has a secrete parameter $K_{T T P}$ which is shared between the tag and the TTP. This parameter is expected to be known only by the tag and the $T T P$, even not a legitimate owner. Now, we present an attack to retrieve this parameter by a legitimate old owner. In this attack, the old owner at the first generates the table $T B$ and for $0 \leq x<2^{16}$ stores $(x, P R N G(x))$ in $T B$. Next, assume that the current secret shared between the tag $T$ and the owner is $K_{M}^{1}$ and the $T T P$ wants that all rights over the tag $T$ are transferred to the reader $R_{I D 2}$ from the reader $R_{I D 1}$. The attack procedure which is performed by the reader $R_{I D 1}$ is as follows:

1) In the complete ownership transfer phase of the protocol, the TTP generates a random number $r_{4}^{1}$ and updates $K_{M}^{1}(i)$ as $K_{M}^{2}(i)=P R N G\left(\operatorname{Per}\left(K_{M}^{1}(i), r_{4}^{1}(i)\right)\right.$ for $i=1, \ldots, 6$.

2) The TTP calculates $H^{1}(i)=r_{4}^{1}(i) \oplus$ $P R N G\left(K_{T T P}(i)\right)$ and $L^{1}(i)=P R N G\left(K_{M}^{1}(i) \oplus\right.$ $\left.r_{4}^{1}(i)\right)$ for $i=1, \ldots, 6$ and sends $K_{M}^{2}$ to the reader $R_{I D 2}$ (the new owner of the tag $T$ ) and $H^{1}$ and $L^{1}$ to the tag $T$.

3) The old owner $R_{I D 1}$, as the adversary, eavesdrops $H^{1}$ and $L^{1}$.

4) Given the eavesdropped $H^{1}$ and $L^{1}$ and the table $T B$, the old owner $R_{I D 1}$ does as follows for all $i=1$ to 6 :

- Computes $r_{4}^{1}(i)=P R N G^{-1}\left(L^{1}(i)\right) \oplus K_{M}^{1}(i)$;

- Assigns $H^{1}(i) \oplus r_{4}^{1}(i)$ to $P R N G\left(K_{T T P}(i)\right)$ and calculates $K_{T T P}(i)$ by looking up at $T B$.

Following the above passive attack, the old owner retrieves $r_{4}^{1}(i)$ and $K_{T T P}(i)$ for $i=1, \ldots, 6$. Since $K_{T T P}$ is the value that is needed as the permanent parameter to access the $\operatorname{tag} T$, the old owner $R_{I D 1}$ finds a permanent control like $T T P$ on the tag $T$ in this attack. On the other hand, the old owner $R_{I D 1}$ knows $K_{M}^{1}$ and it can calculate $K_{M}^{2}(i)=$ $\operatorname{PRNG}\left(\operatorname{Per}\left(K_{M}^{1}(i), r_{4}^{1}(i)\right)\right)$ which is the secret parameter shared between the tag $T$ and the new owner $R_{I D 2}$. This information compromises the new owner privacy.

The complexity of the given attack is eavesdropping a sessions between the target tag and the new owner. The proposed attack is simulated experimentally. The success probability of the attacker is almost 1 for 1000 runs of the attack.

Now we present another attack that an adversary can follow to extract $K_{M}$ and $P R N G\left(K_{T T P}\right)$. Similarly, as the off-line phase of the attack, the adversary generates the table $T B$ and for $0 \leq x<2^{16}$ stores $(x, P R N G(x))$ in $T B$. Then, the adversary does as follows:

1) In complete ownership transfer phase of the protocol, the TTP generates a random number $r_{4}^{1}$ and updates $K_{M}^{1}(i)$ as $K_{M}^{2}(i)=P R N G\left(\operatorname{Per}\left(K_{M}(i), r_{4}^{1}(i)\right)\right.$ for $i=1, \ldots, 6$.

2) The TTP calculates $H^{1}(i)=r_{4}^{1}(i) \oplus$ $P R N G\left(K_{T T P}(i)\right)$ and $L^{1}(i)=P R N G\left(K_{M}^{1}(i) \oplus\right.$ $\left.r_{4}^{1}(i)\right)$ for $i=1, \ldots, 6$ and sends $K_{M}^{2}$ to the reader $R_{I D 2}$ (the new owner of the tag $T$ ) and $H^{1}$ and $L^{1}$ to the tag.

3) The adversary eavesdrops and blocks $H^{1}$ and $L^{1}$.

4) The tag $T$ will not authenticate the new owner $R_{I D 2}$ and the TTP generates another random number $r_{4}^{2}$ and updates $K_{M}^{1}(i)$ as $K_{M}^{3}(i)=P R N G\left(\operatorname{Per}\left(K_{M}^{1}(i), r_{4}^{2}(i)\right)\right.$.

5) The TTP calculates $H^{2}(i)=r_{4}^{2}(i) \oplus$ $P R N G\left(K_{T T P}(i)\right)$ and $L^{2}(i)=P R N G\left(K_{M}^{1}(i) \oplus\right.$ $\left.r_{4}^{2}(i)\right)$ for $i=1, \ldots, 6$ and sends $K_{M}^{3}$ to the new owner $R_{I D 2}$ and $H^{2}$ and $L^{2}$ to the $\operatorname{tag} T$. 
6) The adversary eavesdrops $H^{2}$ and $L^{2}$ and does as follows for all $i=1$ to 6 :

a) For $j=1, \ldots, 2^{16}$ does as follows:

- $r_{4}^{1}(i) \longleftarrow j$;

- $P R N G\left(K_{T T P}(i)\right) \longleftarrow H^{1}(i) \oplus r_{4}^{1}(i)$;

- $K_{M}^{1}(i) \longleftarrow P R N G^{-1}\left(L^{1}(i)\right) \oplus r_{4}^{1}(i)$;

- $r_{4}^{2}(i) \longleftarrow P R N G^{-1}\left(L^{2}(i)\right) \oplus K_{M}^{1}(i)$;

- If $H^{2}(i)=r_{4}^{2}(i) \oplus P R N G\left(K_{T T P}(i)\right)$, return $K_{M}^{1}(i)$ and $P R N G^{-1}\left(P R N G\left(K_{T T P}(i)\right)\right)$

Following the above attack, the adversary retrieves $r_{4}^{1}(i)$, $r_{4}^{2}(i), K_{M}^{1}(i)$ and $K_{T T P}(i)$ for $i=1, \ldots, 6$. Since $K_{T T P}$ is the value that is needed as the permanent parameter to access the tag $T$, the adversary $R_{I D 1}$ finds a permanent control like TTP on the tag $T$ in this attack. On the other hand, the adversary extracted $K_{M}^{1}$ and $r_{4}^{2}$ and she can calculate $K_{M}^{3}(i)=\operatorname{PRNG}\left(\operatorname{Per}\left(K_{M}^{1}(i), r_{4}^{2}(i)\right)\right.$ which is the secret parameter shared between the tag $T$ and the new owner $R_{I D 2}$. This information compromises the new owner privacy.

The complexity of the given attack is eavesdropping two sessions between the target tag and the new owner and blocking one session. The proposed attack is simulated experimentally. The success probability of the attacker is almost 1 for 1000 runs of the attack.

\section{DESYNCHRONIZATION ATTACKS ON NiU et al. AUTHENTICATION AND MANAGEMENT PROTOCOL}

In this section, we explain two different desynchronization attacks against Niu et al. authentication and management protocol on mutual authentication phase and ownership delegation phase.

\section{A. Desynchronization attack on mutual authentication phase}

As explained in Section II, in the mutual authentication phase of the Niu et al. protocol, the reader $R_{I D 1}$ generates two random numbers $r n d_{1}$ and $r n d_{2}$ and sends $A, B$ and $C$ to the $\operatorname{tag} T$. Once the $\operatorname{tag} T$ received the messages $A, B$ and $C$, it verifies the received values, updates $K$ and $I D S$ to $K^{*}$ and $I D S^{*}$ respectively and sends $D$ to the reader. In addition, the designers stated that [11, p. 4, Sec. II. B ] "both the reader and the tag should maintain a copy of the old key and IDS to avoid desynchronization problems". Now we present a desynchronization attack which works even with this assumption.

We use $(A, B, C)_{r_{1}^{j}, r_{2}^{j}}^{K^{l}}$ and $(D)_{r_{1}^{j}, r_{2}^{j}}^{K^{l}}$ to show the messages based on the secret key $K=K^{l}$ and random values $r n d_{l}=r_{l}^{j}$ and $r n d_{2}=r_{2}^{j}$. The procedure of the proposed attack is as follows:

1) Assume that the current state of the tag $T$ and the reader $R_{I D 1}$ is $\left(K^{0}, I D S^{0}, K^{1}, I D S^{1}\right)$ where $K^{0}$ and $I D S^{0}$ are the old key and pseudonym as well as $K^{1}$ and $I D S^{1}$ are the current key and pseudonym of the tag $T$.

2) In the next mutual authentication phase, the reader $R_{I D 1}$ sends $(A, B, C)_{r_{1}^{1}, r_{2}^{1}}^{K_{1}^{1}}$ to the $\operatorname{tag} T$.

3) The tag $T$ updates its state to $\left(K^{1}, I D S^{1}, K^{2}, I D S^{2}\right)$ and sends $(D)_{r_{1}^{1}, r_{2}^{1}}^{K^{2}}$ to the reader $R_{I D 1}$.
4) The adversary blocks $(D)_{r_{1}^{1}, r_{2}^{1}}^{K^{2}}$

5) Since the reader $R_{I D 1}$ does not receive the tag's feedback, it will assume that the tag $T$ does not recognize $K^{1}$ and sends $(A, B, C)_{r_{1}^{2}, r_{2}^{2}}^{K^{0}}$ to the tag $T$.

6) However, the tag $T$ has no record of $K^{0}$ and will not authenticate the reader $R_{I D 1}$ any more and the $\operatorname{tag} T$ and the reader $R_{I D 1}$ has been desynchronized.

One may argue that the reader $R_{I D 1}$ will try with $K^{1}$ once again. In this case, the adversary does as follows:

1) Assume that the current secrets of the $\operatorname{tag} T$ and the reader $R_{I D 1}$ is $\left(K^{0}, I D S^{0}, K^{1}, I D S^{1}\right)$.

2) In the next mutual authentication phase, the reader $R_{I D 1}$ sends $(A, B, C)_{r_{1}^{1}, r_{2}^{1}}^{K^{1}}$ to the tag $T$.

3) The tag $T$ updates its states to $\left(K^{1}, I D S^{1}, K^{2}, I D S^{2}\right)$ and sends $(D)_{r_{1}^{1}, r_{2}^{1}}^{K^{2}}$ to the reader $R_{I D 1}$.

4) The adversary stores $(A, B, C)_{r_{1}^{1}, r_{2}^{1}}^{K^{1}}$.

5) The reader $R_{I D 1}$ also updates its state to $\left(K^{1}, I D S^{1}\right.$, $\left.K^{2}, I D S^{2}\right)$.

6) Since the designers stated that $[11$, p. 3, Sec. II. A ] "before either delegation or complete ownership transfer take place, mutual authentication is needed to verify the authority of all parties involved". So, the adversary blocks all the messages of the phase after authentication which can be delegation phase or complete ownership transfer phase. So once again the mutual authentication phase starts.

7) In the next mutual authentication phase, the reader $R_{I D 1}$ sends $(A, B, C)_{r_{1}^{2}, r_{2}^{2}}^{K^{2}}$ to the tag $T$.

8) The adversary stores $(A, B, C)_{r_{1}^{2}, r_{2}^{2}}^{K^{2}}$ and prevents the tag $T$ to receive them.

9) Since the reader $R_{I D 1}$ does not receive the tag's feedback, it will assume that the tag $T$ does not recognize $K^{2}$. So, The reader $R_{I D 1}$ sends $(A, B, C)_{r_{1}^{3}, r_{2}^{3}}^{K^{1}}$ to the $\operatorname{tag} T$.

10) The tag $T$ updates its state to $\left(K^{1}, I D S^{1}, K^{3}, I D S^{3}\right)$ and sends $(D)_{r_{1}^{3}, r_{2}^{3}}^{K^{3}}$ to the reader $R_{I D 1}$.

11) The reader $R_{I D 1}$ also updates its state to $\left(K^{1}, I D S^{1}\right.$, $\left.K^{3}, I D S^{3}\right)$.

12) The adversary sends $(A, B, C)_{r_{1}^{1}, r_{2}^{1}}^{K^{1}}$ to the tag $T$.

13) The tag $T$ sends $(D)_{r_{1}^{1}, r_{2}^{1}}^{K^{2}}$ to the expected reader and also updates its state to $\left(K^{1}, I D S^{1}, K^{2}, I D S^{2}\right)$.

14) The adversary prevents the reader $R_{I D 1}$ to receive $(D)_{r_{1}^{1}, r_{2}^{1}}^{K^{2}}$

15) The adversary sends $(A, B, C)_{r_{1}^{2}, r_{2}^{2}}^{K^{2}}$ to the tag $T$.

16) The tag $T$ sends $(D)_{r_{1}^{1}, r_{2}^{1}}^{K^{4}}$ to the expected reader and also updates its state to $\left(K^{2}, I D S^{2}, K^{4}, I D S^{4}\right)$.

17) The adversary prevents the reader $R_{I D 1}$ to receive $(D)_{r_{1}^{1}, r_{2}^{1}}^{K^{4}}$

After the above attack, the reader has $K^{1}, I D S^{1}, K^{3}, I D S^{3}$ as its records of secret parameters while the tag $T$ has $K^{2}, I D S^{2}, K^{4}$ and $I D S^{4}$. It is clear that, after the given attack neither of the tag's records for secret parameters matches the reader's records for secret parameters. Hence, the tag and the reader have been desynchronized. Although it may be possible to contact the trusted third party(TTP) 
to re-synchronize the tag, but this attack shows that the given protocol does not satisfy the designers expectation. The complexity of the attack is a few runs of the protocol while the success probability is almost 1 .

\section{B. Desynchronization attack on ownership delegation phase}

In the ownership delegation phase of the protocol, The old owner $R_{I D 1}$ sends ticket, EPC,IDS and $K$ through a secure channel to the new owner (the reader $R_{I D 2}$ ). The reader $R_{I D 2}$ generates a random number $r n d_{3}$ and computes $E$ and $F$ using $K$ and $r n d_{3}$. Then, the reader $R_{I D 2}$ sends $E$ and $F$ to the tag $T$. Once the $\operatorname{tag} T$ received the messages $E$ and $F$, it verifies the received values and updates $K$ and $I D S$ to $K^{*}$ and $I D S^{*}$ using $r n d_{3}$. Then, the tag computes $G$ using $K^{*}$ and $I D S^{*}$ and sends it to the reader $R_{I D 2}$.

In the protocol, the parameter $G(i)$ for $i=1, \cdots, 6$ is not dependent on $K^{*}(i), I D S^{*}(i)$ and the random number $r n d_{3}(i)$ selected by the new owner $R_{I D 2}$. It is computed only using the secret key $K(i)$ as shown as follows:

$$
\begin{aligned}
G(i) & =\operatorname{PRNG}\left(K^{*}(i) \oplus I D S^{*}(i)\right) \\
& =\operatorname{PRNG}\left(\operatorname{Per}\left(\operatorname{rnd}_{3}(i), K(i)\right) \oplus K((i+1) \bmod 6)\right. \\
& \left.\oplus \operatorname{Per}\left(\operatorname{rnd}_{3}(i), K(i)\right) \oplus K(i)\right) \\
& =\operatorname{PRNG}(K((i+1) \bmod 6) \oplus K(i)) ;
\end{aligned}
$$

In other word, the response of $E$ and $F$ which are computed by $r n d_{3}$ and $K$, i.e, $G$, is computed by $K$ not $K^{*}$ and $r n d_{3}$. Now, we show that this property can be used by the attacker to perform desynchronization attack in the ownership delegation phase of the protocol. We use $(E, F)_{r_{3}^{j}}^{K^{j}}$ and $(G)^{K^{j}}$ to show the messages based on the secret key $K^{3}$ and the random value $r n d_{3}=r_{3}^{j}$. We assume that both parties are synchronized in state $\left(K^{1}, I D S^{1}\right)$. The procedure of the proposed attack is as follows:

1) To update the tag $T$ by the new owner (the reader $R_{I D 2}$ ) for the first time, the new owner sends $(E, F)_{r_{3}^{1}}^{K^{1}}$ to the $\operatorname{tag} T$.

2) The tag $T$ updates its states to $\left(K^{2}, I D S^{2}\right)$ where $K^{2}(i)=\operatorname{Per}\left(r_{3}^{1}(i), K^{1}(i)\right) \oplus K^{1}((i+1) \bmod 6)$ and $I D S^{2}(i)=\operatorname{Per}\left(r_{3}^{1}(i), K^{1}(i)\right) \oplus K^{1}(i)$. Then, the tag $T$ sends $(G)^{K^{1}}$ to the reader $R_{I D 2}$.

3) The adversary prevents the reader $R_{I D 2}$ to receive $(G)^{K^{1}}$ and stores it.

4) Since the reader $R_{I D 2}$ does not receive the tag's feedback, it will assume that the tag $T$ does not receive $(E, F)_{r_{3}^{1}}^{K^{1}}$. Therefore, the reader $R_{I D 2}$ chooses another random number $r_{3}^{2}$ and sends $(E, F)_{r_{3}^{2}}^{K^{1}}$ to the tag $T$ again.

5) The adversary prevents the tag $T$ to receive $(E, F)_{r_{3}^{2}}^{K^{1}}$ and sends $(G)^{K^{1}}$ (which is stored by the adversary in step 3) to the reader $R_{I D 2}$.

6) Note that according to the property $G$ which is not dependent on the random number selected by the reader, the reader $R_{I D 2}$ detects the validity of $(G)^{K^{1}}$. So, the reader $R_{I D 2}$ updates its state to $\left(K^{3}, I D S^{3}\right)$ where

$$
\begin{aligned}
& K^{3}(i)=\operatorname{Per}\left(r_{3}^{2}(i), K^{1}(i)\right) \oplus K^{1}((i+1) \bmod 6) \text { and } \\
& I D S^{3}(i)=\operatorname{Per}\left(r_{3}^{2}(i), K^{1}(i)\right) \oplus K^{1}(i) .
\end{aligned}
$$

After the above attack, the reader $R_{I D 2}$ has $K^{2}$ and $I D S^{2}$ as its records of secret parameters while the tag $T$ has $K^{3}$ and $I D S^{3}$. It is clear that, these two states are not the same and the adversary succeeds in performing desynchronization attack between the tag $T$ and the new owner $R_{I D 2}$. Although it may be possible to contact the old owner $R_{I D 1}$ to re-synchronize the tag, but this attack shows that the given protocol does not satisfy the designers expectation. The complexity of the attack is only three runs of the protocol and the probability of a successful de-synchronization attack is equal to 1 .

\section{CONClusion}

In this paper, we scrutinized the security of the mutual authentication and ownership transfer management protocol proposed by Niu et al. Precisely, we present secret disclosure and desynchronization attacks against the protocol with the complexity of a few runs of the protocol and the success probability of almost 1 . This paper shows that the need to secure EPC-C1G2 complaint protocols is still unmet and the new secure protocols must be designed.

\section{REFERENCES}

[1] G. Avoine and X. Carpent. Yet another ultralightweight authentication protocol that is broken. In J. Hoepman and I. Verbauwhede, editors, RFIDSec 2012, volume 7739 of Lecture Notes in Computer Science, pages 20-30. Springer, 2013.

[2] G. Avoine, X. Carpent, and B. Martin. Privacy-friendly synchronized ultralightweight authentication protocols in the storm. J. Network and Computer Applications, 35(2):826-843, 2012.

[3] N. Bagheri and M. Safkhani. Secret disclosure attack on kazahaya, a yoking-proof for low-cost RFID tags. IACR Cryptology ePrint Archive, 2013:453, 2013

[4] N. Bagheri, M. Safkhani, P. Peris-Lopez, and J. E. Tapiador. Weaknesses in a new ultralightweight RFID authentication protocol with permutation - RAPP. Security and Communication Networks, 7(6):945-949, 2014.

[5] H.-Y. Chien. Sasi: A new ultralightweight RFID authentication protocol providing strong authentication and strong integrity. IEEE Trans. Dependable Sec. Comput., 4(4):337-340, 2007.

[6] Class-1 generation 2 UHF air interface protocol standard version 1.2.0, Gen2, 2008. http://www.epcglobalinc.org/standards/.

[7] P. D'Arco and A. D. Santis. Weaknesses in a recent ultra-lightweight rfid authentication protocol. In S. Vaudenay, editor, AFRICACRYPT, volume 5023 of Lecture Notes in Computer Science, pages 27-39. Springer, 2008.

[8] P. D'Arco and A. D. Santis. On ultralightweight RFID authentication protocols. IEEE Trans. Dependable Sec. Comput., 8(4):548-563, 2011.

[9] R. Doss, W. Zhou, and S. Yu. Secure RFID tag ownership transfer based on quadratic residues. IEEE Transactions on Information Forensics and Security, 8(2):390-401, 2013.

[10] A. Falahati and H. Jannati. All-or-nothing approach to protect a distance bounding protocol against terrorist fraud attack for low-cost devices. Electronic Commerce Research, 15(1):75-95, 2015.

[11] S. J. Haifeng Niu, Eyad Taqieddin. EPC Gen2v2 RFID Standard Authentication and Ownership Management Protocol . IEEE Transactions on Mobile Computing, ?(?):?, 2014.

[12] Information technologyRadio frequency identification for item management. Part 6:parameters for air interface communications at $860 \mathrm{MHz}$ to $960 \mathrm{MHz}$.http://www.iso.org. 2005.

[13] H. Jannati and A. Falahati. Cryptanalysis and enhancement of a secure group ownership transfer protocol for RFID tags. In C. K. Georgiadis, H. Jahankhani, E. Pimenidis, R. Bashroush, and A. Al-Nemrat, editors, ICGS3/e-Democracy 2011, volume 99 of Lecture Notes of the Institute for Computer Sciences, Social Informatics and Telecommunications Engineering, pages 186-193. Springer, 2011. 
[14] H. Liu, H. Ning, Y. Zhang, D. He, Q. Xiong, and L. T. Yang. Groupingproofs-based authentication protocol for distributed RFID systems. IEEE Trans. Parallel Distrib. Syst., 24(7):1321-1330, 2013.

[15] S. B. Miles, S. E. Sarma, and J. R. Williams. RFID technology and applications. Cambridge University Press, 2011.

[16] B. Niu, X. Zhu, H. Chi, and H. Li. Privacy and authentication protocol for mobile RFID systems. Wireless Personal Communications, 77(3):1713-1731, 2014

[17] P. Peris-Lopez, J. C. H. Castro, J. M. Estévez-Tapiador, and A. Ribagorda. Emap: An efficient mutual-authentication protocol for low-cost RFID tags. In R. Meersman, Z. Tari, and P. Herrero, editors, OTM Workshops (1), volume 4277 of Lecture Notes in Computer Science, pages 352-361. Springer, 2006.

[18] P. Peris-Lopez, J. C. H. Castro, J. M. Estévez-Tapiador, and A. Ribagorda. Advances in ultralightweight cryptography for low-cost rfid tags: Gossamer protocol. In WISA, pages 56-68, 2008.

[19] P. Peris-Lopez, J. C. Hernandez-Castro, J. M. Estevez-Tapiador, and A. Ribagorda. Lmap: A real lightwight mutual authentication protocol for low-cost rid tags. In Proceedings of RFIDSec06 Workshop on RFID Security, Graz,Austria, 12-14 July 2006.

[20] P. Peris-Lopez, A. Orfila, J. C. Hernandez-Castro, and J. C. A. van der Lubbe. Flaws on RFID grouping-proofs. guidelines for future sound protocols. J. Network and Computer Applications, 34(3):833-845, 2011.

[21] P. Peris-Lopez, A. Orfila, A. Mitrokotsa, and J. C. A. van der Lubbe. A comprehensive RFID solution to enhance inpatient medication safety. $I$. J. Medical Informatics, 80(1):13-24, 2011.

[22] R. C.-W. Phan. Cryptanalysis of a new ultralightweight RFID authentication protocol - sasi. IEEE Transactions on Dependable and Secure Computing, 6(4):316-320, 2009.

[23] M. Safkhani, N. Bagheri, and M. Naderi. Strengthening the security of EPC C-1 G-2 RFID standard. Wireless Personal Communications, 72(2):1295-1308, 2013

[24] M. Safkhani, N. Bagheri, and M. Naderi. A note on the security of isrfid, an inpatient medication safety. I. J. Medical Informatics, 83(1):8285, 2014.

[25] M. Safkhani, P. Peris-Lopez, N. Bagheri, M. Naderi, and J. C. H Castro. On the security of tan et al. serverless RFID authentication and search protocols. In J. Hoepman and I. Verbauwhede, editors, RFIDSec 2012, volume 7739 of Lecture Notes in Computer Science, pages 1-19. Springer, 2013.

[26] S. Sundaresan, R. Doss, S. Piramuthu, and W. Zhou. A robust grouping proof protocol for RFID EPC C1G2 tags. IEEE Transactions on Information Forensics and Security, 9(6):961-975, 2014.

[27] S. Sundaresan, R. Doss, S. Piramuthu, and W. Zhou. Secure tag search in RFID systems using mobile readers. IEEE Trans. Dependable Sec. Comput., 12(2):230-242, 2015.

[28] C. C. Tan, B. Sheng, and Q. Li. Secure and serverless RFID authentication and search protocols. IEEE Transactions on Wireless Communications, 7(4):1400-1407, 2008.

[29] Y. Tian, G. Chen, and J. Li. A new ultralightweight RFID authentication protocol with permutation. IEEE Communications Letters, 16(5):702$705,2012$.

[30] A. Wickboldt and S. Piramuthu. Patient safety through RFID: vulnerabilities in recently proposed grouping protocols. J. Medical Systems, 36(2):431-435, 2012. 